http://jmscr.igmpublication.org/home/ ISSN (e)-2347-176x ISSN (p) 2455-0450 crossref DOI: https://dx.doi.org/10.18535/jmscr/v9i1.43 \\ Journal Of Medical Science And Clinical Research \\ An official Publication of IGM Publication
}

\title{
Phenotypic Characterization and Antimicrobial Susceptibility Pattern of Enterococcal Isolates from Clinical Samples with Special Reference to Vancomycin Minimum Inhibitory Concentration (MIC)
}

\author{
Authors \\ Saba Shama ${ }^{1}$, Anuradha De ${ }^{2}$, Jayanthi Shastri ${ }^{3}$ \\ Department of Microbiology, T. N. Medical College \& B.Y.L Nair Charitable Hospital, Mumbai, India \\ Corresponding Author

\section{Saba Shama} \\ Department of Microbiology, T. N. Medical College \& B.Y.L Nair Charitable Hospital, Mumbai, India
}

\begin{abstract}
Introduction: Enterococcal infections pose therapeutic challenges because of its intrinsic and acquired resistance to vancomycin as well as the ability to produce high level gentamicin resistance (HLGR) and $\beta$ lactamases.

Objectives: A study was undertaken to characterize different strains of enterococci from clinical specimens and to study their antimicrobial susceptibility pattern, including Minimum Inhibitory Concentration (MIC) to vancomycin, high-level aminoglycoside resistance (HLAR) and $\beta$-lactamase production.

Material and Methods: Single enterococcal isolates recovered from clinical specimens received for bacterial culture in this hospital during January 2016 to June 2017 were included in the study. Enterococci were identified and speciated by standard biochemical tests. The isolated enterococci were then tested for antimicrobial susceptibility by Kirby Bauer Disc Diffusion Method (KBDDM), as per CLSI guidelines. Susceptibility to vancomycin was also performed by Minimum Inhibitory Concentration (MIC) using E-strip method. HLAR was detected by using gentamicin disc $120 \mu \mathrm{g}$ and $\beta$-lactamase production by nitrocefin disc.

Results: Total 100 enterococci were isolated during this period, out of which 90 were Enterococcus faecalis, 8 E. faecium and 2 E. solitarius. Most of the samples were from pus and wound swabs (49\%). Maximum isolation was from Medicine ward (25\%), followed by Surgery ward (14\%). Among ICUs, maximum isolation was from MICU (13\%). Antibiotic susceptibility pattern of enterococcal isolates showed Vancomycin resistance in 3\% isolates by KBDDM and in 4\% isolates by MIC. Maximum resistance was shown against erythromycin (64.7\%). High level gentamycin resistance (HLGR) was seen in $48 \%$ isolates and $13 \%$ isolates were $\beta$-lactamase producers.

Conclusion: This study highlighted the problem of multi-drug resistant enterococci, especially with reference to VRE and HLAR. Therefore, an effective infection control policy is required to control the spread of enterococci, with increasing awareness amongst microbiologists \& clinicians.
\end{abstract}

Keywords: Enterococcus species; ABS pattern; Vancomycin MIC.

\section{Introduction}

Enterococci are the most common aerobic and facultative anaerobic gram-positive cocci that occur singly, in pairs or in short chains. These form an indigenous flora of the intestinal tract, oral cavity and the genitourinary tract of the humans and animals. Enterococci, once regarded by many as harmless commensals, have emerged as one of the 
most important nosocomial pathogens worldwide and are associated with high mortality ${ }^{[1]-[3]}$.

The main sites of colonization of enterococcus in the hospitalized patients are soft tissue wounds, ulcers, infections of the gastrointestinal tract, urinary tract infections (UTIs) and bacteraemia. Urinary tract instrumentation or catheterization, genitourinary pathology, prior use of antibiotics, mechanical ventilation, intravenous lines and prolonged hospitalization are some of the predisposing factors for enterococcal infections ${ }^{[2], ~[3] ~}$ Enterococci not only show intrinsic resistance to many antibiotics, they readily acquire resistance genes and are also capable of transferring resistance genes to other bacteria, like Staphylococcus aureus ${ }^{[3]}$. They are intrinsically resistant to cell wall active agents like penicillin, ampicillin if used alone and are also inherently resistant to other antibiotics such as cephalosporins and aminoglycosides ${ }^{[2],[4]}$. Resistance to high concentration of aminoglycosides, usually mediated by aminoglycoside modifying enzymes, is widespread and gradually increasing among enterococci ${ }^{[3],[4]}$.

Vancomycin resistant enterococci (VRE) have been reported worldwide. Enterococcal infections pose therapeutic challenges because of its intrinsic and acquired resistance to vancomycin as well as the ability to produce $\beta$-lactamases. Considering the increasing frequency of VRE isolation from different parts of India, appropriate surveillance and continuous monitoring is very important to control the spread and cross contamination of these resistant clones in hospital settings mostly originating from undetected VRE carriers or contaminated environment or via contaminated gloves/gowns of Health Care Workers (HCWs). Hence increasing trends in VRE strains is a cause of concern ${ }^{[2],[4]}$. Prevalence of VRE ranges from $1 \%$ to $23.07 \%$ in India. The overall national data of enterococcal infection rate is yet to be available from India due to paucity of cumulative data collection system ${ }^{[5]-[10]}$. Maximum papers on VRE are from North India. As there are few data available about VRE from this part of India, this study was undertaken to characterize different strains of enterococci isolated from clinical specimens, to study their antimicrobial susceptibility pattern including high-level aminoglycoside resistance, vancomycin resistance, $\beta$-lactamase production and Minimum Inhibitory Concentration (MIC) to vancomycin.

\section{Materials and Methods}

It was a prospective study conducted in a tertiary care hospital in Mumbai during a period of $1 \frac{1}{2}$ years (from January 2016 to June 2017). Single enterococcal isolates recovered from clinical specimens, including urine, pus, blood culture, respiratory samples, body fluids, etc. received for bacterial culture was included in the study.

The samples received in the laboratory from outpatients and inpatients were collected. Enterococci were identified and speciated by standard biochemical tests ${ }^{[11]}$.

Antimicrobial susceptibility of enterococci were then performed on Mueller Hinton agar by Kirby Bauer Disc Diffusion Method (KBDDM), according to CLSI guidelines ${ }^{[12]}$. Antibiotics used were penicillin, ampicillin, vancomycin, linezolid, teicoplanin and erythromycin (except for urinary isolates). For urinary isolates, norfloxacin and nitrofurantoin were used in addition to the other antibiotics. Susceptibility to vancomycin was performed also by using E-strip method (HiMedia) for detecting Minimum Inhibitory Concentration $(\mathrm{MIC})^{[12]}$. Based on resistance pattern to vancomycin and teicoplanin, Van A and Van B phenotypes were identified $^{[11]}$.

HLGR was determined by using gentamicin $120 \mu \mathrm{g}$ disc (HiMedia) and $\beta$-lactamase production was determined by using nitrocefin disc (Hardy Diagnostics $)^{[12]}$.

\section{Results}

Out of the total 100 enterococcal strains isolated, 90 were Enterococcus faecalis, 8 were E. faecium and 2 were E. solitarius. Figure 1 shows the arrangement of enterococci in primary smear, colonies of enterococci on blood agar, a strain of VRE on Mueller Hinton agar by vancomycin E-strip and antibiotic susceptibility pattern of enterococcus 


\section{JMSCR Vol||09||Issue||01||Page 232-239||January}

by KBDDM. Most of the samples were from pus and wound swabs (49), followed by urine (32) and blood culture (13). Other sites were high vaginal swab (3), tracheal secretions (1), ascitic fluid (1) and central line tip (1).

Majority of the isolates (96\%) were from indoor patients. Out of 100, males comprised $48 \%$ and females 52\%, with Male: Female ratio being 0.92:1. Out of total isolation, $64 \%$ of enterococci were from wards and 32\% were from Intensive Care Units. Maximum isolation was from Medicine ward (25\%), followed by Surgery ward (14\%). Among ICUs, maximum isolation was from MICU (13\%).

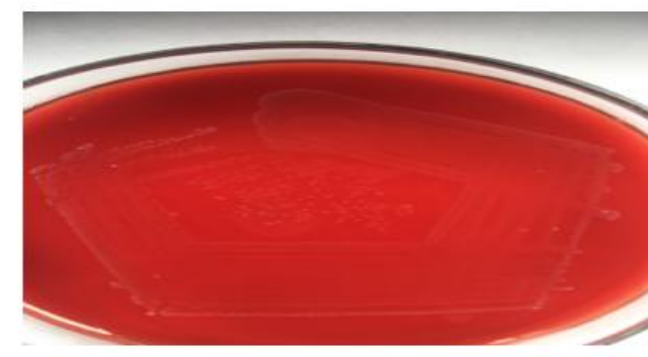

Growth of Enterococcus species on Blood agar

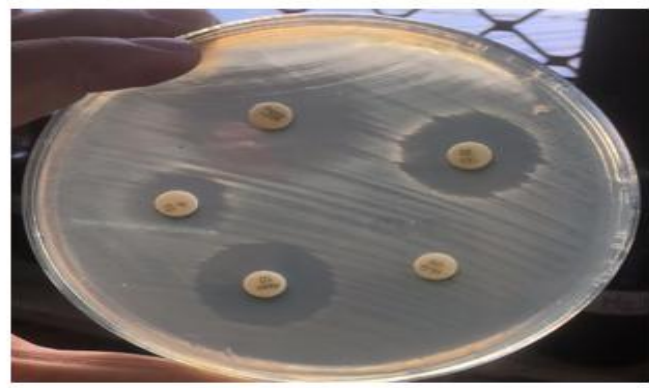

ABS of enterococcus on MHA- $S$ to Ampicillin, Linezolid \& Teicoplanin : $R$ to Penicillin \& HLG

Fig 1. Smear, Growth and ABS pattern of enterococci
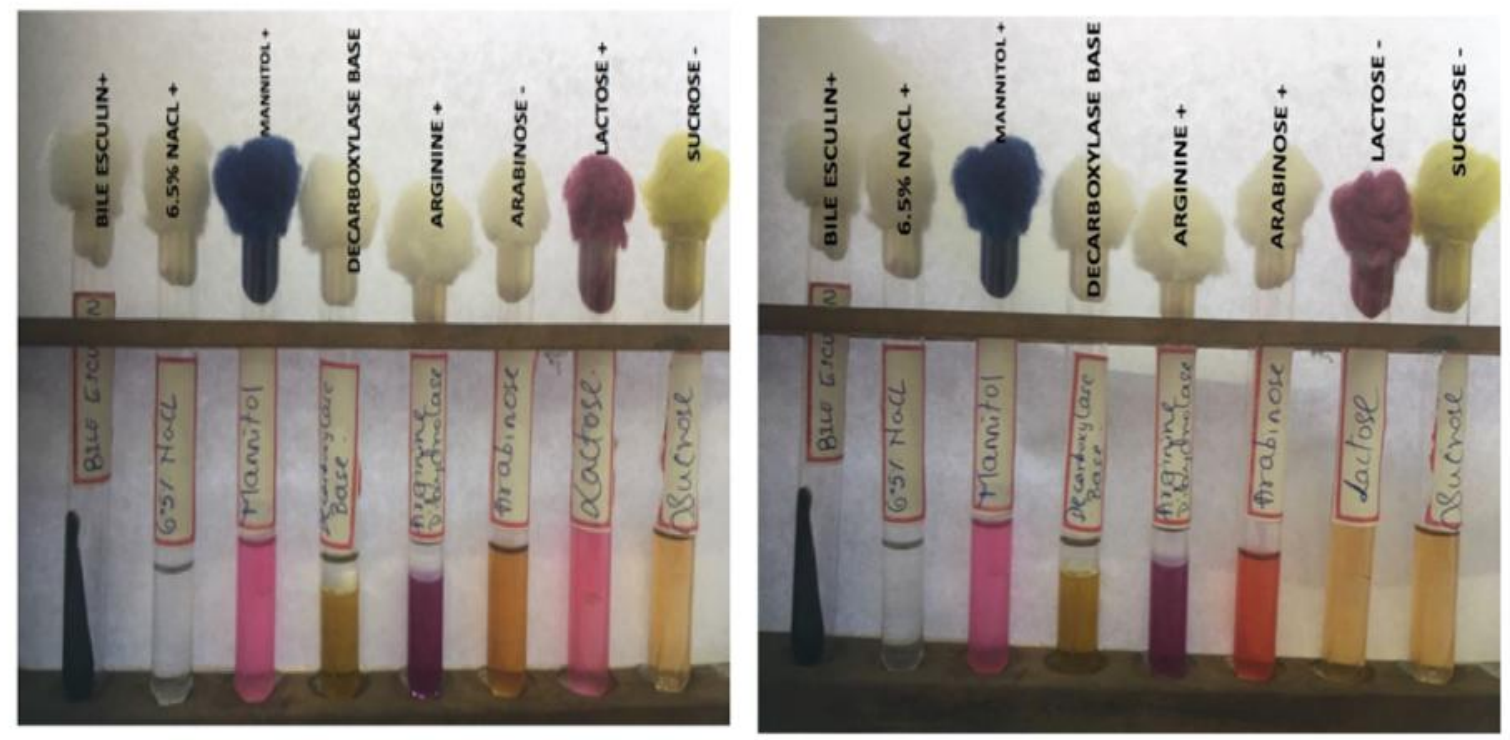

Biochemical reactions of $E$. faecalis. Biochemical reactions of $E$. faecium

Fig 2. Biochemical reactions of Enterococcus species 


\section{JMSCR Vol||09||Issue||01||Page 232-239||January}

Figure 2 shows the biochemical properties of $E$. faecalis \& E. faecium. Among the 49 isolates from pus/wound, $89.8 \%$ was E. faecalis and $10.2 \%$ was $E$. faecium. Among 32 urinary isolates, $90.6 \%$ was $E$. faecalis, $3.1 \%$ E. faecium and $6.3 \%$ E. solitarius. From 13 isolates from blood culture, $84.6 \%$ was $E$. faecalis and $15.4 \%$ was E. faecium.

Table 1 shows overall antibiotic resistance pattern of enterococci isolated in this study. Vancomycin resistance was seen in 3\% isolates by KBDDM and in $4 \%$ isolates by MIC. Maximum resistance was shown by erythromycin (64.7\%), followed by $30 \%$ to penicillin \& $22 \%$ to ampicillin. For urinary isolates, $53.1 \%$ were resistant to norfloxacin whereas only $25 \%$ were resistant to nitrofurantoin. All the isolates were susceptible to Linezolid (100\%) and Teicoplanin susceptibility was $97 \%$.

High level gentamycin resistance (HLGR) was seen in $48 \%$ isolates, of which $89.6 \%$ (43/48) were $E$. faecalis and $10.4 \%(5 / 48)$ were E. faecium. In the present study, $13 \%$ isolates were $\beta$-lactamase producers, of which E. faecalis was $61.5 \%(8 / 13)$ and $38.5 \%(5 / 13)$ was E. faecium. Both the $E$. solitarius did not show high level gentamicin resistance and did not produce $\beta$-lactamase.

Table 1: Overall antibiotic resistant pattern of Enterococcus species

\begin{tabular}{|c|c|c|c|c|}
\hline Antibiotics & $\begin{array}{c}\text { E. faecalis } \\
n=90(\%)\end{array}$ & $\begin{array}{c}\text { E. faecium } \\
n=8(\%)\end{array}$ & $\begin{array}{c}\text { E. } \\
\text { solitarius } \\
n=2(\%)\end{array}$ & $\begin{array}{c}\text { Total } \\
\text { n=100 (\%) }\end{array}$ \\
\hline Penicillin & $23(25.5)$ & $06(75)$ & $01(50)$ & $30(30)$ \\
\hline Ampicillin & $16(17.7)$ & $06(75)$ & 00 & $22(22)$ \\
\hline $\begin{array}{l}\text { Vancomycin } \\
\text { KBDDM }\end{array}$ & $01(1.1)$ & $02(25)$ & 00 & $03(3)$ \\
\hline Vancomycin MIC & $01(1.1)$ & $03(37.5)$ & 00 & $04(4)$ \\
\hline Linezolid & 00 & 00 & 00 & 00 \\
\hline Teicoplanin & $01(1.1)$ & $02(25)$ & 00 & $03(3)$ \\
\hline Erythromycin (68)* & $39(63.9)$ & $05(71.4)$ & - & $44(64.7)$ \\
\hline Norfloxacin (32)** & $16(55.1)$ & $01(100)$ & 00 & $17(53.1)$ \\
\hline Nitrofurantoin (32)** & $07(24.1)$ & $01(100)$ & 00 & $08(25)$ \\
\hline
\end{tabular}

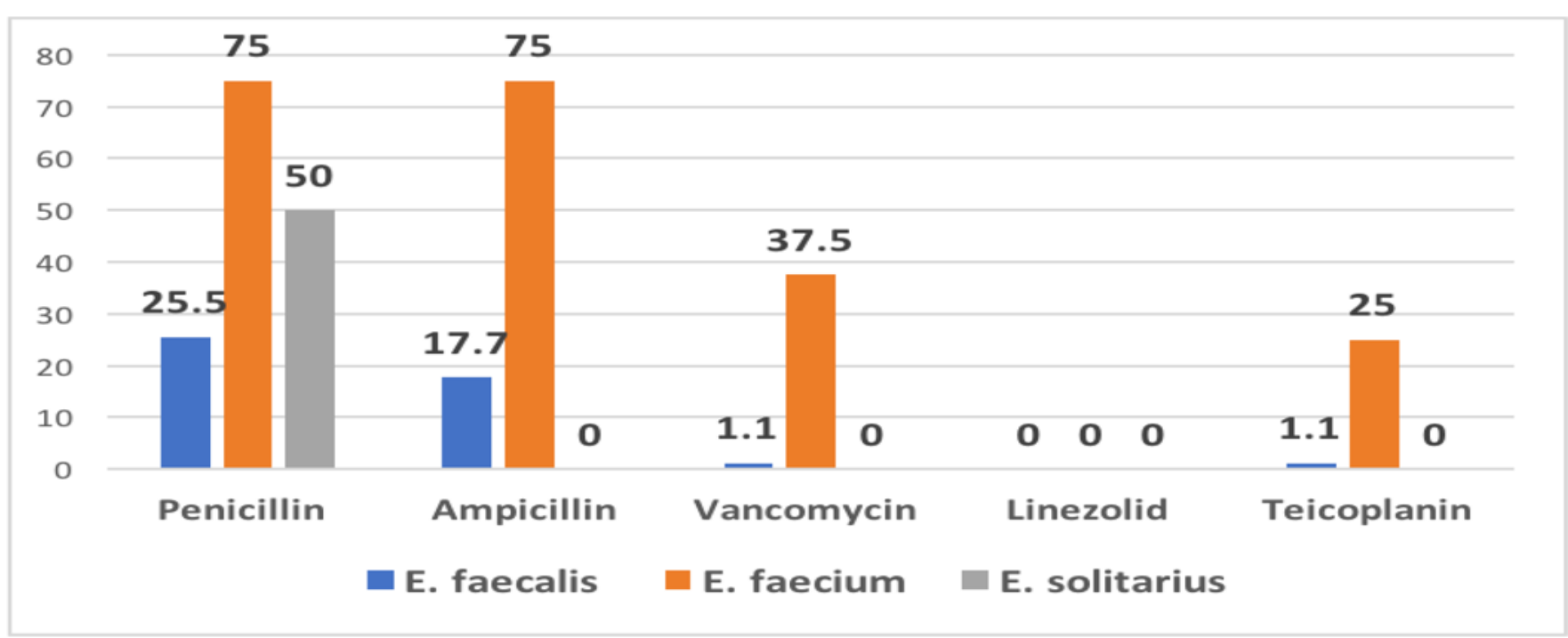

Fig 3: Percentage antibiotic resistance pattern of enterococcal isolates to five antibiotics 
Fig 3 shows percentage antibiotic resistance pattern of different enterococcal isolates to five antibiotics. E. faecium $75 \%$ antibiotic resistance to penicillin and ampicillin. Antibiotic resistance to Vancomycin and Teicoplanin was also $37.5 \%$ and $25 \%$ respectively in E. faecium. Resistance to all antibiotics was much less in E. faecalis as compared to E. faecium.

\section{Discussion}

In the last four decades, has emerged as an important pathogen, not only because of their ability to cause serious infections like endocarditis, bacteraemia and urinary tract infections but also because of their increasing resistance to many antimicrobial agents. The emergence of Vancomycin Resistant Enterococci (VRE) and their rapid spread worldwide, has made it extremely difficult to treat serious enterococcal infections, leaving the clinicians with very few therapeutic options.

Commonest species isolated in this study was $E$. faecalis (90\%), followed by E. faecium (8\%) and $E$. solitarius (2\%). Percentage of E. faecalis and $E$. faecium isolated in the present study was exactly similar to that of Gordon et al $(90 \%$ and $8 \%$ respectively) ${ }^{[6]}$. In the present study, overall penicillin resistance was $30 \%$ (Table1), which is close to the study reported by Miskeen et al $(23.13 \%)^{[7]}$.

Urinary isolates of enterococci were resistant to norfloxacin (53.1\%), whereas resistance to nitrofurantoin was $25 \%$ (Table 1). This is in acceptance to the study by Rajesh et $\mathrm{al}^{[8]}{ }^{[\mathrm{l}}$ who have reported $25 \%$ resistance to norfloxacin and only $4 \%$ resistance to nitrofurantoin for urinary isolates.

$\beta$-lactamase production was seen in $62.5 \%$ isolates of E. faecium and $8.8 \%$ isolates of E. faecalis. Gordon et $\mathrm{al}^{[6]}$ have reported $1.6 \% \quad \beta$-lactamase producing isolates.

High level gentamycin resistance (HLGR) was seen in $48 \%$ isolates in the present study, of which $89.6 \%$ were among E. faecalis and $10.4 \%$ among $E$. faecium. This is almost similar to the study by
Mendiratta et $\mathrm{al}^{[9]}$, who reported $46 \%$ HLGR. Oberoi et $\mathrm{al}^{[13]}$ reported HLGR in $42.1 \%$ enterococcal isolates, where HLGR among $E$. faecium was higher $(56.16 \%)$ than in E. faecalis $(29.45 \%)$. Lall et $\mathrm{al}^{[14]}$ reported slightly higher percentage of HLGR $(60.5 \%)$

In the present study, VRE was encountered in $4 \%$ isolates which corroborates with a study by Rajesh et $\mathrm{a}^{[8]}$ who has reported $5.7 \%$ VRE. In the present study, MIC of 4 strains of enterococci was found to be $>8 \mu \mathrm{g} / \mathrm{ml}$, giving an overall vancomycin resistance of $4 \%$. One of these four strains was reported sensitive by KBDDM. So KBDDM detected only three strains as resistant to vancomycin (Table 1). Errors were determined by comparing the two methods and this turned out to be a very major error. Thus, the correlation between the two methods was poor. This finding is supported by Taneja et al ${ }^{[10]}$ from Chandigarh, who missed four out of eight strains of vancomycin resistance by KBDDM. This study therefore highlights the limitation of KBDDM in detecting vancomycin resistance.

Prevalence of VRE in various Indian studies ranges from $1 \%$ to $23 \%$ (Table 2). VRE with community acquired sources should be detected early, as this will limit the spread of VRE in the hospital environment. Since colonized patients leave the hospital environment, the possibility that transmission might occur in the community cannot be discounted. If transmission of VRE from unrecognized sources can be identified and controlled, colonization of hospitalized patients will be reduced, leading to lower rates of nosocomial infection due to $\mathrm{VRE}^{[20]}$.

Van A is the commonest phenotype in India (Table 2). Van B phenotype is the second most common phenotype of VRE reported worldwide. In the present study, out of three VRE in E. faecium, two were Van A \& one Van B and the single VRE in $E$. faecalis was of Van A phenotype 
Table 2: Comparison of VRE isolation with other studies

\begin{tabular}{|l|c|c|c|c|c|c|}
\hline Study & Year & $\begin{array}{c}\text { Total } \\
\text { samples }\end{array}$ & $\begin{array}{c}\text { VRE } \\
\text { No. (\%) }\end{array}$ & Samples pos VRE & Phenotype & $\begin{array}{c}\text { MIC } \\
\text { range }\end{array}$ \\
\hline Mathur et al[15] & 2003 & 444 & $5(1)$ & Blood (3), urine (1), soft tissue (1) & $\begin{array}{c}\text { Van A (4) } \\
\text { Van B (1) }\end{array}$ & $26-512$ \\
\hline Karmarkar et al[16] & 2004 & 52 & $12(23.07)$ & Urine, blood, pus* & Van B & $>8$ \\
\hline Taneja et al[10] & 2004 & 144 & $8(5.5)$ & Urine (8) & $\begin{array}{c}\text { Van B (6) } \\
\text { Van C (2) }\end{array}$ & $8-32$ \\
\hline De et al[17] & 2009 & 200 & $2(1)$ & Foley's tip (1), ascitic fluid (1) & Van A & 128 \\
\hline Mulla et al[18] & 2011 & 92 & $8(8)$ & Urine (5), blood (2), CSF (1) & Van B & $8-32$ \\
\hline Praharaj et al[19] & 2013 & 367 & $32(8.7)$ & $\begin{array}{c}\text { Pus/wound (7), } \\
\text { Urine (6), blood (4), CFS (3), } \\
\text { synovial fluid*, peritoneal fluid* }\end{array}$ & $\begin{array}{c}\text { Van A (28) } \\
\text { Van B (4) }\end{array}$ & $8-128$ \\
\hline Present study & 2017 & 100 & $4(4)$ & Urine (2), pus (1), blood (1) & $\begin{array}{c}\text { Van A (3), } \\
\text { Van B (1) }\end{array}$ & $32-64$ \\
\hline
\end{tabular}

*Sample size not mentioned

Mortality in VRE infection in this study was $50 \%$ (2/4) and both were E. faecium. Our study is almost similar to the study by Bhavnani et $\mathrm{al}^{[11]}$, who reported 52\% mortality in VRE patients.

Surveillance of family members of recently discharged patients known to be colonized or infected with VRE, should be done. A VRE case should be considered to carry the pathogen indefinitely, unless 3 consecutive cultures, at least one week apart, taken after antibiotic treatment, from stool or rectal swabs are negative for VRE. Once these negative cultures are documented, the individual can be considered VRE-free and special infection control practices may be terminated ${ }^{[20]}$.

As enterococcus is ubiquitous and constitute a large reservoir of resistant genes, awareness amongst Microbiologists and Clinicians should be created about the spread of vancomycin resistance in Staphylococcus aureus ${ }^{[21]}$.

Linezolid susceptibility of $100 \%$ in the present study was in accordance with the study by Shah et $\mathrm{al}^{[18]}$ and Narayanaswamy et $\mathrm{al}^{[22]}$. However, there are few exceptions.

Deshpande et $\mathrm{al}^{[23]}$ and Rajesh et $\mathrm{al}^{[7]}$ have reported $2.4 \%$ and $2 \%$ linezolid resistance respectively in enterococcal isolates ${ }^{[8],[22]}$. A case report of linezolid resistant enterococcus from blood culture of an adult patient has been reported by Baveja et $\mathrm{al}^{[24]}$ from Mumbai.

\section{Conclusion}

This study emphasizes the screening for VRE of all enterococci isolated from different clinical samples. Detection of MIC for vancomycin can be performed by E-strip test, as Macro broth dilution method is a laborious procedure. All laboratories should have effective methods for detection of vancomycin resistance accurately and continuously monitor the same. It will be helpful in reducing the morbidity and mortality due to VRE, in hospitalized patients. Therefore, it is recommended that MIC of enterococcus for vancomycin should be carried out on routine basis, especially whenever enterococci are isolated from seriously ill patients. Surveillance of the community to detect the reservoirs of VRE should also be done from time to time.

Detection of high-level aminoglycoside resistance (HLAR) in enterococcal isolates should also be made mandatory in all laboratories.

The present study also highlighted the problem of multidrug resistant enterococci. Therefore, an effective prevention and control against the spread of MDR enterococci requires coordinated efforts from different areas of hospital and this can only be achieved by prudent use of antibiotics, education of all cadres of hospital staff about the problems of drug resistance, early detection and reporting of isolates in hospitalized patients and in hospital environment. Whenever there is a rise in MDR enterococci, immediate implementation of appropriate infection control measures is need of the day. 


\section{References}

1. Murray BE. The life and times of the enterococcus. Clin Microbiol Rev 1990;3(1):46-65.

2. Cetinkaya Y, Falk P, Mayhall CG. Vancomycin-Resistant Enterococci. Clin Microbiol Rev 2000;13(4):686-707.

3. Moellering RC. Vancomycin-Resistant Enterococci. Clin Infect Dis 1998;26:119699.

4. Murray BE. Vancomycin-Resistant Enterococci. Amer J Med 1997;102(3):28493.

5. Gordon S, Swenson JM, Hill BC, Facklam R, Cooksey RC, Jarvis W TenoverF. Antimicrobial Susceptibility Patterns of common and unusual species of Enterococcus.

ClinMicrobiol1992;30:2373-78.

6. Miskeen A. P, Deodhar L. Antimicrobial Susceptibility pattern of Enterococcus species from Urinary Tract Infections. J Assoc Phys India 2002;50:378-81.

7. Rajesh S, Subathra N, Neelaveni D, Nirmala S. Detection of High Level Aminoglycoside Resistance and Vancomycin Resistance in Enterococcus Species Isolated from Various Clinical Samples of Tertiary Care Medical College Hospital. Int J CurrMicrobiolAppl Sci 2017;6(5):2731-9.

8. Mendiratta DK, Kaur H, Deotale V, Thamke DC, Narang R, Narang P. Status of high level aminoglycoside resistant Enterococcus faecium and Enterococcus faecalis in a rural hospital of central India. Indian $\mathbf{J}$ Med Microbiol 2008;26(4):369.

9. Taneja N, Rani P, Emmanuel R, Sharma M. Significance of vancomycin resistant enterococci from urinary specimens at a tertiary care centre in northern India. Indian Journal of Medical Research. 2004;119(2):72.

10. Bhavnani SM, Drake JA, Forrest A, Deinhart JA, Jones RN, Biedenbach DJ, Ballow $\mathrm{CH}$, National Nosocomial
Resistance Surveillance Group. A nationwide, multicentre, case-control study comparing risk factors, treatment, and outcome for vancomycin-resistant and susceptible enterococcal bacteraemia. Diagnostic Microbiol Infect Dis 2000;36(3):145-58.

11. Koneman EW, Allen SD, Janda WM, Schreckenberger PC, Winn WC Jr. The gram-positive cocci. Part II: Streptococci, enterococci and the Streptococcus-like bacteria. In Koneman's Colour Atlas and Textbook of Diagnostic Microbiology. $5^{\text {th }}$ Ed. Philadelphia, Lippincott. 1997;pp 577-650.

12. Clinical and Laboratory Standards Institute (CLSI). Performance Standards for Antimicrobial Susceptibility Testing. 26th Ed. CLSI supplement M100S (ISBN 156238-923-8), 2016; pp 82-85.

13. Oberoi L, Aggarwal A. Multidrug resistant enterococci in a rural tertiary care hospital-a cause of concern. JK Science. 2010;12(3):157.

14. Lall N, Basak S. High Level Aminoglycoside Resistant Enterococcus species: A Study. Int J Curr Res Rev 2014;6(3):16.

15. Mathur P, Kapil A, Chandra R, Sharma P DB. Antimicrobial resistance in Enterococcus faecalis at a tertiary care center of northern India. Indian J Med Res 2003;118:25-8.

16. Karmarkar MG, Gershom ES, Mehta PR. Enterococcal infections with special reference to phenotypic characterization \& drug resistance. Indian Journal of Medical Research. 2004;119:22-5.

17. De A, Bindlish A, Kumar S, Mathur M. Vancomycin resistant enterococci in a tertiary care hospital in Mumbai. Indian $\mathbf{J}$ Med Microbiol 2009; 27:375-6.

18. Shah L, Mulla S, Patel KG, Rewadiwala S. Prevalence of enterococci with higher resistance level in a tertiary care hospital: a 
matter of concern. Natl J Med Res 2012;2(1):25-7.

19. Praharaj I, Sujatha S, Chandra Parija S. Phenotypic and genotypic characterization of vancomycin resistant enterococcus isolates from clinical samples. Indian J Med Res 2013;138:549-56.

20. McDonald LC, Kuehnert MJ, Tenover FC, Jarvis WR. Vancomycin-resistant enterococci outside the health-care setting: prevalence, sources, and public health implications. Emerging infectious diseases. 1997;3(3):311.

21. Leclercq R. Enterococci acquire new kinds of resistance. Clinical Infectious Diseases. 1997;24(Supplement 1):S80-4.

22. Narayanaswamy A, Rajalakshmi K, Varadharajan M. Speciation and antimicrobial susceptibility pattern of Enterococci from a tertiary health care center of south India. J Pharm Res. 2011;4(4):989-90.

23. Deshpande VR, Karmarkar MG, Mehta PR. Prevalence of multi-drug resistant enterococci in a tertiary care hospital in Mumbai, India J Infect 2013;7(02):155-8.

24. Baveja SM, De AS, Talwadekar SL, Vasave RA. Linezolid Resistant Enterococcus (LRE) faecium from blood culture of an adult burn patient: First report of LRE from Mumbai. J Global Infect Dis 2017;9(1):31. 